\title{
Genetic diversity and population structure of rice landraces from Eastern and North Eastern States of India
}

Basabdatta Das ${ }^{1 \dagger}$, Samik Sengupta ${ }^{2 \dagger}$, Swarup Kumar Parida ${ }^{4}$, Bipasha Roy ${ }^{1}$, Mrityunjay Ghosh ${ }^{3}$, Manoj Prasad ${ }^{4}$ and Tapas Kumar Ghose ${ }^{1 *}$

\begin{abstract}
Background: Adaptations to different habitats across the globe and consequent genetic variation within rice have resulted in more than 120,000 diverse accessions including landraces, which are vital genetic resources for agronomic and quality traits. In India the rice landraces of the states West Bengal, Assam, Mizoram, Manipur and Nagaland are worthy candidates for genetic assessment. Keeping the above in view, the present study was conducted with the aim to (i) calculate the genetic distances among the accessions of 83 landraces collected from these states along with 8 check accessions (total 91 accessions) using 23 previously mapped SSR markers and (ii) examine the population structure among the accessions using model-based clustering approach.

Results: Among the 91 accessions, 182 alleles were identified which included 51 rare and 27 null alleles. The average PIC value was $0.7467 /$ marker. The non-aromatic landraces from West Bengal was most diverse with 154 alleles and an average PIC value of 0.8005/marker, followed by the aromatic landraces from West Bengal with 118 alleles and an average PIC value of 0.6524/marker, while the landraces from North East ranked third with 113 alleles and an average PIC value of $0.5745 /$ marker. In the dendrogram distinct clusters consisting of predominantly aromatic landraces and predominantly North East Indian landraces were observed. The non-aromatic landraces from West Bengal were interspersed within these two clusters. The accessions were moderately structured, showing four sub-populations (A-D) with an Fst value of $0.398,0.364,0.206$ and 0.281 , respectively. The assigned clustering of accessions was well in agreement in both distance-based and model-based approaches.
\end{abstract}

Conclusions: Each of the accessions could be identified unequivocally by the SSR profiles. Genetically the non aromatic landraces from West Bengal were most diverse followed by the aromatic landraces from the same state. The North Eastern accessions ranked third. Further, grouping of accessions based on their agronomic traits may serve as a resource for future studies, leading to the improvement of rice. Moreover in-situ preservation of the landraces is also a means of protection of biodiversity and cultural heritage.

Keywords: Oryza sativa, Landrace, Genetic diversity, SSR polymorphism, Population structure

\footnotetext{
* Correspondence: tapasghoselab@gmail.com

${ }^{\dagger}$ Equal contributors

'Division of Plant Biology, Bose Institute, Main Campus, 93/1 A.P.C. Road,

700009 Kolkata, West Bengal, India

Full list of author information is available at the end of the article
} 


\section{Background}

Rice (Oryza sativa L.) is a major ingredient in cuisines world over, in the form of breakfast cereals, staple carbohydrate, snacks, alcoholic beverage and desserts. In addition to the two major subspecies, japonica and indica, [1] several other minor rice types have been identified with genetic markers $[2,3]$ which include the upland droughttolerant Aus germplasms of India and Bangladesh, the deep-water Ashinas of Bangladesh, and the aromatic Basmati rice of India. As a consequence of adaptations to different habitats, extensive genotypic and phenotypic diversity exists within $O$. sativa, resulting in about 120,000 different accessions [4]. These accessions range from traditional rice landraces preserved by indigenous farmers to the commercially bred cultivars developed during the green revolution. According to Harlan [5] landraces are "balanced populations in equilibrium with both the environment and pathogens, and are genetically dynamic". They are local varieties of a domesticated plant species which were adapted to the natural and cultural environment in which they live. Each landrace has particular properties or characteristics; early maturity, adaptation to particular soil types, resistance or tolerance to biotic and abiotic stresses, and in the expected end usage of the grains. India is home to many such landraces and the ones from the state of West Bengal and North Eastern States of the country are especially diverse morphologically and genetically, and are worthy candidates for detailed examination. This study estimates a broad overview of Simple Sequence Repeat (SSR) based genetic diversity present in 83 different rice landrace selected from the wide array available in the states of West Bengal, Assam, Nagaland, Mizoram and Manipur using 23 SSR markers.

The topography of the state of West Bengal and the seven North Eastern States of India namely, Arunachal Pradesh, Assam, Manipur, Meghalaya, Mizoram, Nagaland and Tripura, is one of nature's marvels which has brought in proximity the snow capped peaks of the Himalayas, the ecological hot spots of the North Eastern foothills, the Brahmaputra valley, the fertile Gangetic plain, and the estuarine regions of the Sunderban delta. High rainfall, humidity, varied topography and altitude, heavy natural selection pressures of diseases and pests, introductions over time and space from adjoining countries, introgression from the wild and weedy relatives, tribal preferences and environmental stresses have made the region rich both in floristic and crop diversities [6].

Molecular markers, especially DNA-based markers, have been used extensively for the study of genetic diversity, unambiguous identification of germplasm and their protection under the trade related intellectual property rights (TRIPS) of the World Trade Organization (WTO). Mackill [7] classified 134 japonica rice cultivars (both traditional and modern) using
RAPD and was successful in separating the temperate and tropical japonica genotypes. Glaszmann [8] quantified the genetic diversity of 289 rice cultivars from various parts of Northeast India at 14 isozyme loci and was able to segregate the cultivars into the varietal groups I to VI. The gene diversity index [9] for that study was 0.341 for Northeast Indian rice which was close to that calculated for all Asian rice (0.346), and thus very high considering the small size of the area under survey. SSR or Simple Sequence Length Polymorphism (SSLP) markers have been used for evaluating rice genetic diversity by a number of groups [10-14] for estimating variation between the indica and japonica subspecies [15] and classifying Oryza sativa L. genotypes [16]. Blair et al., [17] differentiated indica and japonica rice genotypes using Inter Simple Sequence Repeat (ISSR) polymorphism data. ISSR polymorphism has also been used for genetic diversity and phylogenetic analysis in 42 genotypes including 17 wild Oryza species [18]. ISSR and SSR markers have been used to study genetic diversity of Oryza nivara Sharma et Shastry, genotypes collected from different geographical regions [19]. Herrera et al. [20] used 48 simple-sequence-repeat (SSR) markers to assess the genetic diversity of 11 Venezuelan rice cultivars released by the National Rice Breeding Program between 1978 and 2007 and detected 203 alleles. Pervaiz et al., [21] used 35 SSR markers to detect genetic diversity in 75 rice landraces and identified 142 alleles. The polymorphism information content (PIC) ranged from 0.124 to 0.836 , with an average of 0.569. Maytinee et al., [22] used InDel (Insertion/Deletion), ISSR and SSR markers to detect genetic diversity among 126 Thai rice accessions. Behera et al., [23] used 36 microsatellite markers to assess genetic diversity in a set of 33 medicinal rice genotypes and detected 166 polymorphic loci. The PIC values ranged between 0.24 and 0.956 with an average of 0.811 per locus. However a thorough SSR based genetic diversity analysis, of the landraces of West Bengal and North Eastern States is yet to be undertaken.

Previous workers like Ge et al., [24] have reported the linkage of SSR loci on rice chromosome 2 with cooked kernel expansion and the same for chromosome 3 with width expansion of rice kernels. Quantitative Trait Loci (QTL) mapping of rice by Bai et al., [25] have indicated the linkage of rice grain shape (grain length/ grain breadth ratio) to SSR loci RM112 and RM530 on chromosome 2. Ahmadi and Fotokian [26] have indicated the linkage of markers RM251 and RM282 on rice chromosome 3 to potassium ion concertration. Lin et al., [27] have shown linkage of RM112 and RM530 on chromosome 2 to leaf rolling in rice plants due to water stress. QTL mapping of rice indicated the linkage of rice grain and kernel length, breadth, length/breadth ratio kernel length after cooking and aroma to 18 different SSR markers [28,29]. The set of markers used in 
this study to assess genetic diversity included 18 markers from the references above along with 5 more (a total of 23 markers) with linkage to Sheath blight and Blast resistance [30] HSP 80 gene [31] and Beta amylase gene. Only the most popular landraces that are regularly cultivated by small farming communities were chosen for this study. Each of the landraces had some special characteristic like aroma, disease resistance, yield etc for which they have been cultivated by farmers down the ages. Keeping the above in view, the present study was conducted with the aim to (i) calculate the genetic distances among 91 rice germplasm using 23 mapped SSR markers; and (ii) examine the population structure among the accessions using model-based clustering approach.

\section{Methods}

\section{Plant materials}

A total of 91 rice germplasms including 83 rice landraces and 8 check germplasms were collected from rice research stations in India. The landraces were divided in 4 categories; namely 26 aromatic accessions from West Bengal, 31 non-aromatic accessions from West Bengal, 26 accessions from the North Eastern States which included 6 aromatic and 6 non aromatic germplasms from Nagaland, 3 non-aromatic germplasms from Mizoram and 1 accession from Manipur and check germplasms included 2 basmati accessions and 6 high yielding varieties. The name, abbreviation source, category, kernel length, kernel shape, amylose content aroma level and inferred group from population structure analysis, of each rice accession are given in Table 1 and the outline maps in Figure 1 show the states from which the collection was made.

\section{Isolation of genomic DNA and PCR amplification}

Three day-old rice seedlings germinated from 10 well developed grains from a single plant of each genotype were used for genomic DNA isolation according to the method of Walbot [34]. PCR amplification of this DNA was done with 23 pairs of SSR markers. The name, motif, chromosomal location, annealing temperature of the markers, their associated phenotypic trait and corresponding references are given in Table 2. DNA amplification was carried out in $25 \mu \mathrm{l}$ volumes in a MJR thermal cycler (USA). Each reaction mixture contained $1 \mu \mathrm{l}$ of genomic DNA (100 ng), $0.5 \mu \mathrm{l}$ of each primers (at a concentration of $10 \mathrm{pmole} / \mu \mathrm{l}), 2.5 \mu \mathrm{l}$ of $10 \times$ PCR buffer, $0.75 \mu \mathrm{l}$ of $50 \mathrm{mM} \mathrm{MgCl} 2,0.25 \mu \mathrm{l}$ of $2.5 \mathrm{mM}$ dNTP mixture, $0.2 \mu \mathrm{l}$ (1 unit) of 5 unit/ $\mu \mathrm{l}$ Taq DNA polymerase and $19.3 \mu \mathrm{l}$ of PCR-grade water. The temperature profile of the first PCR cycle was $97^{\circ} \mathrm{C}$ for 5 mins, $55-60^{\circ} \mathrm{C}$ (as necessary in accordance to Table 2) for $2 \mathrm{~min}$; followed by 35 cycles of
$1 \mathrm{~min}$ at $95^{\circ} \mathrm{C}, 1 \mathrm{~min}$ at $55-60^{\circ} \mathrm{C}$ and $2 \mathrm{~min}$ at $72^{\circ} \mathrm{C}$. The final extension was at $72^{\circ} \mathrm{C}$ for $10 \mathrm{~min}$.

\section{Polyacrylamide Gel electrophoresis}

The PCR products were resolved in native polyacrylamide gel electrophoresis (PAGE) according to Sambrook et al. [35] in 6\% gel in vertical electrophoresis tank (gel size of $16 \mathrm{~cm} \times 14 \mathrm{~cm}$, Biotech, India) with Tris-Acetate-EDTA buffer at $150 \mathrm{~V}$. The gel was stained with ethidium bromide $(5 \mu \mathrm{g}$ of EtBr in $200 \mathrm{ml}$ of Tris-Borate-EDTA buffer) washed twice with distilled water and analyzed in Gel Documentation System (Biorad, USA).

\section{Allele scoring}

A cluster of two to five discrete bands (stutter) was apparent in the stained gels for most of the markers. The size (in nucleotides) of the most intensely amplified band for each microsatellite marker was determined using the Quantity One software (Biorad, USA), based on the migration of the band relative to standard molecular weight size markers (100 bp DNA ladder SibEnzyme) [36]. IR36 was used as a molecular weight reference in each gel because a sequence-based estimate of allele size in this germplasm is available, as described in Panaud et al., [11]. The band with the lowest molecular weight for each SSR marker was assigned allele number 1 and the progressively heavier bands were assigned incrementally. For the individual markers, the presence of an allele in each of the germplasms was recorded as " 1 " and the absence of an allele was denoted as "0" [36]. A genotype was assigned a null allele for an SSR locus whenever an amplification product(s) was not detected for the particular genotype $\times$ marker combination [37]. When an allele was found in less than $5 \%$ of the germplasms under study, it was designated as rare [38].

\section{Genetic diversity analysis using SSR profiles and bootstrap analysis}

A 1/0 matrix was constructed for each marker using the information of presence or absence of alleles. The resultant matrix was used to calculate genetic similarities among the accessions according to Jaccard's coefficient [39] using NTSYS-pc software package (version 2.02e) [40]. Using pairwise similarity matrix of Jaccard's coefficient, a phylogenetic tree was constructed by the Unweighted PairGroup Method of Arithmetic average (UPGMA) and Neigh bor-Joining (NJoin) module of the NTSYS-pc. Support for clusters was evaluated by bootstrap analysis using WinBoot software [41] through generating 1,000 samples by resampling with replacement of characters with in the combined $1 / 0$ data matrix. 
Table 1 Names, source, category, kernel length, kernel shape, amylose content, aroma and group of the 91 genotypes included in this study

\begin{tabular}{|c|c|c|c|c|c|c|c|}
\hline Genotypes & Source & Category & Kernel length & Kernel shape & Amylose content & Aroma & Group* \\
\hline \multicolumn{8}{|c|}{ Accessions of aromatic genotypes from West Bengal } \\
\hline Badshahbhog & RRS, Chinsurah & WBA & Small & Medium & Low & Fairly strong & B \\
\hline Chinikamini 1 & RRS, Chinsurah & WBA & Small & Round & Low & Moderately strong & B \\
\hline Chinikamini 2 & RRS, Chinsurah & WBA & Medium & Slender & Intermediate & Fairly strong & C \\
\hline Danaguri & RRS, Chinsurah & WBA & Small & Medium & Low & Moderate & B \\
\hline Gobindobhog 1 & RRS, Chinsurah & WBA & Small & Medium & Low & Strong & B \\
\hline Gobindobhog 2 & RRS, Sekhampur & WBA & Small & Medium & Low & Strong & B \\
\hline Gopalbhog & SARF, Kashipur & WBA & Small & Medium & Intermediate & Fairly strong & B \\
\hline Kalogobindobhog & ATC, Fulia & WBA & Medium & Round & Low & Moderate & $\mathrm{D}$ \\
\hline Kalojira & ATC, Fulia & WBA & Small & Bold & Intermediate & Moderately strong & B \\
\hline Kalonunia & SARF, Kashipur & WBA & Small & Medium & Intermediate & Fairly strong & B \\
\hline Kaminibhog & SARF, Kashipur & WBA & Small & Round & Intermediate & Fairly strong & B \\
\hline Kanakchur & SARF, Kashipur & WBA & Small & Round & Low & Moderate & $\mathrm{D}$ \\
\hline Katarihog & RRS, Chinsurah & WBA & Medium & Medium & Low & Fairly strong & A \\
\hline Khasdhan & RRS, Chinsurah & WBA & Small & Medium & Low & Moderately strong & B \\
\hline Lilabati & RRS, Chinsurah & WBA & Small & Bold & Low & Fairly strong & B \\
\hline Mohanbhog & ATC, Fulia & WBA & Small & Bold & Low & Fairly strong & B \\
\hline Narayan bhog & ATC, Fulia & WBA & Long & Slender & Low & Moderate & $D$ \\
\hline Narayan purna & ATC, Fulia & WBA & Small & Round & Low & Fairly strong & $\mathrm{D}$ \\
\hline NC 324 & RRS, Chinsurah & WBA & Medium & Medium & Low & Fairly strong & B \\
\hline NC 365 & RRS, Chinsurah & WBA & Medium & Slender & Low & Fairly strong & B \\
\hline Radhunipgol 1 & RRS, Chinsurah & WBA & Small & Bold & Low & Fairly strong & B \\
\hline Radhunipgol 2 & RRS, Chinsurah & WBA & Small & Bold & Low & Fairly strong & B \\
\hline Radhatilak & RRS, Chinsurah & WBA & Small & Medium & Low & Fairly strong & B \\
\hline Tulaipanji & RRS, Sekhampur & WBA & Small & Medium & Low & Moderately strong & B \\
\hline Tulsibhog & RRS, Sekhampur & WBA & Small & Bold & Intermediate & Moderate & B \\
\hline Tulsimanjari & ATC, Fulia & WBA & Small & Medium & Low & Moderate & B \\
\hline \multicolumn{8}{|c|}{ Accessions of aromatic genotypes from West Bengal } \\
\hline Asitkalma & ATC, Fulia & WBNA & Long & Slender & Intermediate & Nil & $\mathrm{D}$ \\
\hline Bahurupi & ATC Fulia & WBNA & Extra long & Medium & Intermediate & Nil & C \\
\hline Bangalakshmi & ATC Fulia & WBNA & Extra long & Medium & Intermediate & Nil & C \\
\hline Bangladeshi patnai & ATC Fulia & WBNA & Extra long & Slender & Intermediate & Nil & C \\
\hline Bhasamanik & ATC Fulia & WBNA & Extra long & Slender & Intermediate & Nil & $\mathrm{D}$ \\
\hline Chamarmani & ATC, Fulia & WBNA & Extra long & Medium & Intermediate & Nil & C \\
\hline Dudherswar & SARF, Kashipur & WBNA & Medium & Slender & Intermediate & $\mathrm{Nil}$ & C \\
\hline Hatipanjra & RRS, Chinsurah & WBNA & Medium & Slender & Intermediate & Nil & $\mathrm{D}$ \\
\hline Heerasail & RRS, Chinsurah & WBNA & Long & Medium & Intermediate & Nil & $\mathrm{D}$ \\
\hline Kalisankar & ATC, Fulia & WBNA & Small & Medium & Intermediate & Nil & $\mathrm{D}$ \\
\hline kalopahar & RRS, Chinsurah & WBNA & Extra long & Slender & High & Nil & C \\
\hline Katki & ATC, Fulia & WBNA & Medium & Medium & Intermediate & $\mathrm{Nil}$ & $\mathrm{D}$ \\
\hline Kelas & ATC, Fulia & WBNA & Long & Medium & Intermediate & Nil & $\mathrm{D}$ \\
\hline Kele & ATC, Fulia & WBNA & Long & Medium & Intermediate & $\mathrm{Nil}$ & C \\
\hline Kerala sundari & ATC, Fulia & WBNA & Extra long & Slender & Intermediate & Nil & C \\
\hline
\end{tabular}


Table 1 Names, source, category, kernel length, kernel shape, amylose content, aroma and group of the 91 genotypes included in this study (Continued)

\begin{tabular}{|c|c|c|c|c|c|c|}
\hline Khandagiri & RRS, Chinsurah & WBNA & Extra long & Slender & High & Nil \\
\hline Lakshmansail & RRS, Chinsurah & WBNA & Extra long & Slender & Intermediate & Nil \\
\hline Latasail & RRS, Chinsurah & WBNA & Extra long & Medium & Intermediate & Nil \\
\hline Madina & SARF, Kashipur & WBNA & Extra long & Slender & Intermediate & Nil \\
\hline Mahsuri & RRS, Chinsurah & WBNA & Extra long & Slender & High & Nil \\
\hline Marichsail & SARF, Kashipur & WBNA & Small & Medium & Low & Nil \\
\hline Metedhan & SARF, Kashipur & WBNA & Extra long & Medium & Intermediate & Nil \\
\hline Mugai & SARF, Kashipur & WBNA & Medium & Medium & Intermediate & Nil \\
\hline Para & ATC, Fulia & WBNA & Medium & Medium & Low & Nil \\
\hline Parbol & ATC, Fulia & WBNA & Extra long & Medium & High & Nil \\
\hline Paizam & ATC, Fulia & WBNA & Extra long & Slender & High & Nil \\
\hline Raghusail & RRS, Chinsurah & WBNA & Medium & Medium & High & Nil \\
\hline Raniakanda & ATC, Fulia & WBNA & Extra long & Slender & Intermediate & Nil \\
\hline Raspanjar & RRS, Chinsurah & WBNA & Long & Medium & Intermediate & Nil \\
\hline Sadashankar & RRS, Chinsurah & WBNA & Extra long & Slender & High & Nil \\
\hline Talmari & RRS, Chinsurah & WBNA & Extra long & Medium & High & Nil \\
\hline \multicolumn{7}{|c|}{ Accessions from North Eastern States } \\
\hline Aijong & AAU & NA, ASM & Long & Slender & Intermediate & Nil \\
\hline Biroi dhan & NBPGR, Umiam & NA, ASM & Long & Slender & Low & Nil \\
\hline Bhog joha & NBPGR, Umiam & $A R, A S M$ & Medium & Medium & Intermediate & Faint \\
\hline Bhu & NBPGR, Umiam & $N A, M Z$ & Medium & Medium & Intermediate & Nil \\
\hline Boro Chhaiyamora & AAU & NA, ASM & Long & Slender & Intermediate & Nil \\
\hline Buhrimtui & NBPGR, Umiam & NA, MZ & Medium & Medium & Low & Nil \\
\hline Desi Dhan & NBPGR, Umiam & NA, MZ & Medium & Medium & Intermediate & Nil \\
\hline IC524502 & NBPGR, Umiam & NA, NG & Extra long & Medium & Intermediate & Nil \\
\hline IC-524507 & NBPGR, Umiam & $N A, N G$ & Extra long & Medium & Intermediate & Nil \\
\hline IC-311005 & NBPGR, Umiam & $N A, N G$ & Medium & Medium & Intermediate & Nil \\
\hline IC-311003 & NBPGR, Umiam & $N A, N G$ & Extra long & Slender & Low & Nil \\
\hline IC-524526 & NBPGR, Umiam & $N A, N G$ & Medium & Medium & Intermediate & Nil \\
\hline IC-524531 & NBPGR, Umiam & $N A, N G$ & Extra long & Medium & Low & Nil \\
\hline IC-524528 & NBPGR, Umiam & $N A, N G$ & Extra long & Medium & Intermediate & Nil \\
\hline IC-524529 & NBPGR, Umiam & $N A, N G$ & Extra long & Medium & Intermediate & Nil \\
\hline IC-311028 & NBPGR, Umiam & $N A, N G$ & Extra long & Medium & Intermediate & Nil \\
\hline IC-524530 & NBPGR, Umiam & $N A, N G$ & Extra long & Medium & Intermediate & Nil \\
\hline IC360739 & NBPGR, Umiam & NA, ASM & Extra long & Medium & Low & Nil \\
\hline Joha & RRS, Chinsurah & $A R, A S M$ & Extra long & Slender & Intermediate & Moderately strong \\
\hline Kala Boro dhan & NBPGR, Umiam & NA, ASM & Medium & Medium & Very Low & Faint \\
\hline Kachalo & NBPGR, Umiam & NA, ASM & Medium & Medium & Intermediate & $\mathrm{Nil}$ \\
\hline Kalojeera & AAU & $A R, A S M$ & Small & Slender & Intermediate & Strong \\
\hline Lal Binni & AAU & $A R, A S M$ & Extra long & Slender & Very low & Faint \\
\hline Malsara dhan & NBPGR, Umiam & NA, ASM & Extra long & Medium & Low & Nil \\
\hline Morianghou & NBPGR, Umiam & $\mathrm{NA}, \mathrm{MN}$ & Medium & Medium & Intermediate & Nil \\
\hline Prasadbhog & AAU & $A R, A S M$ & Long & Slender & Intermediate & Fairly strong \\
\hline
\end{tabular}


Table 1 Names, source, category, kernel length, kernel shape, amylose content, aroma and group of the 91 genotypes included in this study (Continued)

\begin{tabular}{|c|c|c|c|c|c|c|c|}
\hline \\
\hline $\begin{array}{l}\text { Accessions of check germplasms } \\
\text { IR8 }\end{array}$ & RRS, Chinsurah & ICV & Medium & Medium & Low & $\mathrm{Nil}$ & C \\
\hline IR 36 & RRS, Chinsurah & ICV & Medium & Slender & Intermediate & $\mathrm{Nil}$ & A \\
\hline IR 64 & RRS, Chinsurah & ICV & Extra long & Slender & Intermediate & Nil & C \\
\hline Khitish & RRS, Chinsurah & HYV & Extra long & Slender & Intermediate & Nil & C \\
\hline Lal Swarna & SARF, Kashipur & HYV & Medium & Slender & Intermediate & Nil & D \\
\hline TN 1 & RRS, Chinsurah & HYV & Medium & Slender & Intermediate & $\mathrm{Nil}$ & C \\
\hline Pusa Basmati 1 & RRS, Chinsurah & IA & Extra long & Slender & Intermediate & Strong & C \\
\hline Taraori Basmati & ATC, Fulia & IA & Extra long & Slender & Very Low & Strong & B \\
\hline
\end{tabular}

Abbreviations: WBA Aromatic landrace from West Bengal, WBNA Non aromatic landrace from West Bengal, AR ASM Aromatic landraces from Assam, NA ASM Non aromatic landraces from Assam, NA MN Non aromatic landraces from Manipur, NA MZ Non aromatic landraces from Mizoram, NA NG Non aromatic landraces from Nagaland, ICV International check variety, HYV High yielding variety, AAU Assam Agriculture University, ATC Agricultural training centre, RRS Rice research station, NBPGR National Bureau of Plant Genetic Resources, SARF State agricultural research farm.

*Groups as was determined by STRUCTURE ANALYSIS.

The classification of kernels different kernel lengths and shapes has been done according to Standard Evaluation System (SES, IRRI) [32]. The data of amylose content and aroma was obtained from previous experiments in our laboratory [33].

\section{Calculation of polymorphism information content}

The polymorphism information content (PIC) value modified by Anderson et al., [42] for self pollinated species was used to calculate the PIC value. The formula is as follows:

$$
\mathrm{PIC}_{\mathrm{i}}=1-\sum_{\mathrm{i}=1}^{\mathrm{n}} \mathrm{P}_{\mathrm{ij}}^{2}
$$

where Pij is the frequency of the $j^{\text {th }}$ allele for the $i^{\text {th }}$, SSLP marker.

\section{Population structure analysis}

The association mapping population was analyzed for possible population structure with the model-based program Structure 2.2 [43] for the 91 rice accessions using a length of burn-in period and the number of iterations set at 10000 and a model allowing for admixture and correlated allele frequencies. At least ten runs of Structure were performed by setting the number of sub-populations (K) from $K=2$ to $K=10$. Pair-wise FST values were estimated among four sub-populations using Arlequin [44].

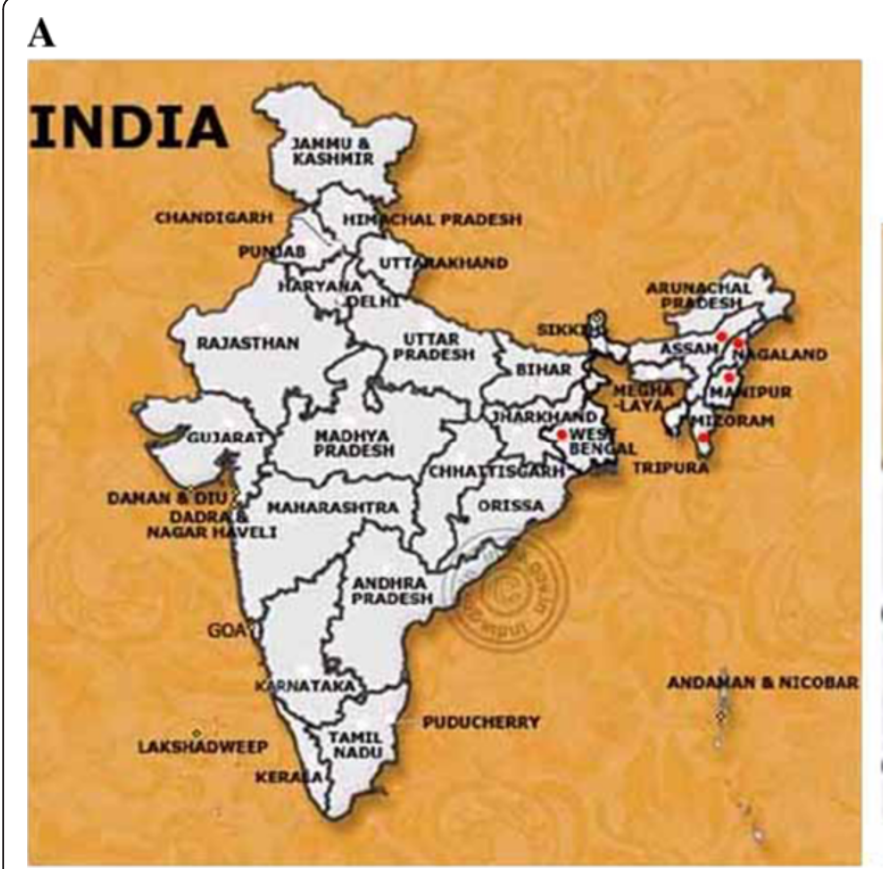

B

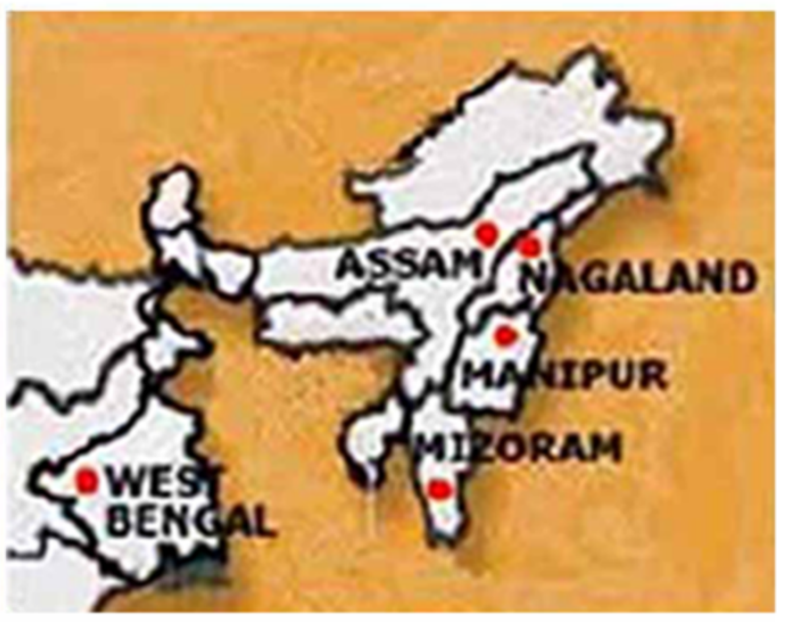

Figure 1 (A) Political map of India showing the states (marked with red dots) from which rice was collected. (B) Enlarged view of the states from which rice was collected. 
Table 2 Name, motif, chromosomal location, annealing temperature and associated phenotypic trait of each SSR marker used for this study

\begin{tabular}{|c|c|c|c|c|c|}
\hline Locus & Motif & Chr* & $T^{* *}$ & Associated phenotypic trait & Reference \\
\hline RM 42 & $(G A) 6$ & 8 & 65 & LAC,CKE,AROMA & Bisaws 2004, Shobha Rani 2006 \\
\hline RM 44 & $(G A) 16$ & 8 & 55 & LAC, L/B AC,CKE & Biswas 2004, Shobha Rani 2006 \\
\hline RM72 & (TAT)5C(ATT)15 & 8 & 55 & Sheath blight resistance & Eizenga et al 2006 \\
\hline RM 80 & (CTT)20 & 8 & 65 & LAC,AROMA & Bisaws 2004, Shobha Rani 2006 \\
\hline RM 112 & $(\mathrm{GAA}) 5$ & 2 & 55 & $\mathrm{GL}, \mathrm{GB}, \mathrm{G} \mathrm{L} / \mathrm{B}, \mathrm{KL}, \mathrm{KB}, \mathrm{K} \mathrm{L} / \mathrm{B}$ & Biswas 2004, Shobha Rani 2006, Bai et al., 2010, Lin et al., 2007 \\
\hline RM 149 & (AT)10 & 8 & 59 & HSP 80 gene & Breusegem et al 1994, Eizenga et al., 2006 \\
\hline RM 152 & (GGC)10 & 8 & 60 & $\mathrm{GL}, \mathrm{GB}, \mathrm{G} L / \mathrm{B}, \mathrm{KL}, \mathrm{KB}, \mathrm{K} \mathrm{L} / \mathrm{B}$ & Biswas 2004, Shobha Rani 2006 \\
\hline RM 182 & (AT)16 & 7 & 59 & Beta amylase gene & Chen, 1993 \\
\hline RM207 & $(G A) 25$ & 2 & 65 & GL,AROMA & Bisaws 2004, Shobha Rani 2006 \\
\hline RM 210 & $(G A) 23$ & 8 & 55 & AROMA & Biswas 2004, \\
\hline RM 218 & $(G A) 24$ & 3 & 55 & $\mathrm{GL}$ & Biswas 2004, Shobha Rani 2006 \\
\hline RM 223 & $(G A) 25$ & 8 & 55 & LAC, L/B AC,CKE & Biswas 2004, Shobha Rani 2006 \\
\hline RM 250 & (CT)17 & 2 & 60 & $\mathrm{GL}, \mathrm{GB}, \mathrm{G} L / \mathrm{B}, \mathrm{KL}, \mathrm{KB}, \mathrm{K} \mathrm{L} / \mathrm{B}$ & Biswas 2004, Shobha Rani 2006 \\
\hline RM 251 & $(\mathrm{CT}) 29$ & 3 & 55 & Sheath blight resistance & Eizenga et al., 2006, Ahmadi and Fotokian 2011 \\
\hline RM 282 & $(G A) 15$ & 3 & 59 & Blast resistance & Eizenga et al., 2006, Ahmadi and Fotokian 2011 \\
\hline RM 284 & $(G A) 8$ & 8 & 55 & LAC, L/B AC,CKE & Biswas 2004, Shobha Rani 2006 \\
\hline RM 310 & (GT)19 & 8 & 55 & $\mathrm{GL}, \mathrm{GB}, \mathrm{G} \mathrm{L} / \mathrm{B}, \mathrm{KL}, \mathrm{KB}, \mathrm{K} \mathrm{L} / \mathrm{B}$ & Biswas 2004, Shobha Rani 2006 \\
\hline RM 337 & (CTT)4-19-(CTT)8 & 8 & 59 & AROMA & Biswas 2004, Shobha Rani 2006 \\
\hline RM 339 & (СТT)8ССТ9ССТ)5 & 8 & 59 & $L A C, L / B A C, C K E$ & Biswas 2004, Shobha Rani 2006 \\
\hline RM 341 & $(\mathrm{CTT}) 20$ & 2 & 55 & Sheath blight resistance & Eizenga et al., 2006 \\
\hline RM 505 & (CT) 12 & 7 & 55 & $\mathrm{GL}, \mathrm{GB}, \mathrm{G} L / \mathrm{B}, \mathrm{KL}, \mathrm{KB}, \mathrm{K} \mathrm{L} / \mathrm{B}$ & Biswas 2004, Shobha Rani 2006 \\
\hline RM 530 & $(G A) 23$ & 2 & 59 & $\mathrm{GL}, \mathrm{GB}, \mathrm{G} L / \mathrm{B}, \mathrm{KL}, \mathrm{KB}, \mathrm{K} \mathrm{L} / \mathrm{B}$ & Biswas 2004, Shobha Rani 2006, Bai et al., 2010, Lin et al., 2007 \\
\hline RM 569 & (CT)16 & 3 & 59 & $\mathrm{GL}, \mathrm{GB}, \mathrm{G} L / B, K L, K B, K \mathrm{~L} / \mathrm{B}$ & Biswas 2004, Shobha Rani 2006 \\
\hline
\end{tabular}

Chr - Rice chromosome T - Annealing temperature.

\section{Results}

\section{Analysis of SSR profiles}

Number of alleles

Table 3 summarizes the analysis of SSR profiles of the 91 rice accessions using 23 SSR markers. The reference molecular weight derived from the standard accession IR-36, for each marker is also showed in Table 3. All the markers showed polymorphism and a total of 182 alleles were identified. The number of alleles ranged from 2 in RM112 to 13 in RM 149. The average number of alleles was 7.9 alleles per locus. Categorically, the average number of alleles for aromatic rice accessions from West Bengal was 5.13 alleles /locus. The SSR profiles of the markers RM42, RM210 and RM530 were the least informative with 3 alleles each, while the most informative marker was RM341 producing 8 alleles. The average number of alleles for the non-aromatic rice accessions from West Bengal was 6.69 alleles /locus. The number of alleles ranged from 4 in the SSR profiles of the markers RM112, RM282 and RM341 to 9 for the markers RM 149, RM223 and RM250. For the germplasm accessions from the North
East Indian States, the average number of alleles was 4.91 alleles /locus. In this group only two alleles were produced by the markers RM112 and RM505, while the highest of 8 alleles was generated by RM149. For the check genotypes the average number of alleles was 2.87 alleles /locus with just 1 allele produced by RM505 and the highest of 4 alleles generated by the markers RM80, RM152, RM210 and RM337.

\section{PIC values}

For all the accessions the PIC values, which denote allelic diversity and frequency, had an average value of 0.747 per marker. The range of PIC value was 0.484 in RM 505 to 0.871 in RM 149. Categorically, average PIC value for aromatic rice from West Bengal was 0.652 per marker with a range of 0.258 in RM 250 to 0.855 in RM 341. For the non aromatic germplasm accessions of West Bengal, the average PIC value was 0.801 per marker and range of PIC value was 0.528 in RM 339 to 0.993 in RM210. The North East Indian set of rice had an average PIC value of 0.575 per marker. The range of PIC value for them was 0.0739 in 
Table 3 Minimum and maximum molecular weight among the alleles, rare alleles (R), null alleles (N) and PIC values for each marker

\begin{tabular}{|c|c|c|c|c|c|c|c|c|c|c|c|c|c|c|c|}
\hline \multirow[t]{2}{*}{ Marker } & \multirow[t]{2}{*}{$\min M W$} & \multirow[t]{2}{*}{$\max \mathrm{MW}$} & \multirow[t]{2}{*}{ IR 36 MW } & \multirow[t]{2}{*}{$\mathbf{R}$} & \multirow[t]{2}{*}{$\mathrm{N}$} & \multicolumn{5}{|c|}{ Number of alleles } & \multicolumn{5}{|c|}{ PIC values } \\
\hline & & & & & & Total & WBA & WBNA & Check & NE & Total & WBA & WBNA & Check & NE \\
\hline RM 42 & 114.39772 & 189.36196 & 166 & 2 & 4 & 9 & 3 & 7 & 3 & 8 & 0.8114962 & 0.5535714 & 0.830385 & 0.625 & 0.8343195 \\
\hline RM 44 & 89.648764 & 109.72365 & 99 & 1 & 1 & 6 & 5 & 6 & 3 & 4 & 0.7793745 & 0.5765306 & 0.817898 & 0.71875 & 0.6272189 \\
\hline RM 72 & 137.82168 & 182.30073 & 166 & 2 & 1 & 7 & 5 & 6 & 3 & 5 & 0.7453206 & 0.5433673 & 0.8022893 & 0.671875 & 0.3934911 \\
\hline RM 80 & 113.39867 & 178.60759 & 142 & 1 & 5 & 7 & 6 & 7 & 4 & 3 & 0.7565511 & 0.7627551 & 0.8605619 & 0.65625 & 0.6005917 \\
\hline RM 112 & 119.83476 & 139.12983 & 128 & 1 & 0 & 4 & 4 & 4 & 3 & 2 & 0.534235 & 0.5331633 & 0.5681582 & 0.40625 & 0.204142 \\
\hline RM 149 & 169.15164 & 277.81621 & 253 & 3 & 0 & 13 & 6 & 9 & 2 & 11 & 0.8701848 & 0.7295918 & 0.9646202 & 0.703125 & 0.8816568 \\
\hline RM 152 & 124.85913 & 159.70665 & 151 & 1 & 1 & 5 & 5 & 6 & 4 & 4 & 0.7045043 & 0.6836735 & 0.7658689 & 0.65625 & 0.3313609 \\
\hline RM 182 & 307.94713 & 372.9556 & 346 & 2 & 0 & 9 & 7 & 7 & 3 & 4 & 0.8246589 & 0.8010204 & 0.8220604 & 0.75 & 0.693787 \\
\hline RM 207 & 64.904795 & 178.32084 & 118 & 4 & 4 & 9 & 6 & 8 & 2 & 7 & 0.7722497 & 0.6887755 & 0.7793965 & 0.375 & 0.8239645 \\
\hline RM 210 & 110.11641 & 218.32062 & 140 & 3 & 2 & 9 & 3 & 9 & 4 & 4 & 0.787103 & 0.6607143 & 0.9927159 & 0.5625 & 0.5325444 \\
\hline RM 218 & 112.20192 & 161.50204 & 148 & 2 & 1 & 8 & 5 & 7 & 3 & 5 & 0.8134283 & 0.6479592 & 0.7887617 & 0.40625 & 0.7869822 \\
\hline RM 223 & 139.79416 & 192.30402 & 165 & 3 & 1 & 9 & 7 & 9 & 3 & 5 & 0.8284024 & 0.7653061 & 0.8647242 & 0.65625 & 0.6390533 \\
\hline RM 250 & 121.09209 & 174.86578 & 153 & 6 & 2 & 9 & 4 & 9 & 3 & 4 & 0.6330153 & 0.2576531 & 0.867846 & 0.53125 & 0.5946746 \\
\hline RM 251 & 104.74847 & 178.47583 & 147 & 1 & 1 & 8 & 5 & 8 & 3 & 5 & 0.779133 & 0.625 & 0.9354839 & 0.8125 & 0.6065089 \\
\hline RM 282 & 122.1457 & 146.14296 & 136 & 2 & 2 & 8 & 4 & 4 & 2 & 6 & 0.8236928 & 0.7270408 & 0.7055151 & 0.421875 & 0.6538462 \\
\hline RM 284 & 129.65555 & 161.73167 & 141 & 3 & 0 & 7 & 5 & 6 & 2 & 5 & 0.7383166 & 0.6505102 & 0.6930281 & 0.21875 & 0.535503 \\
\hline RM 310 & 58.878678 & 126.12462 & 105 & 2 & 1 & 9 & 6 & 7 & 3 & 5 & 0.8030431 & 0.7142857 & 0.8158169 & 0.78125 & 0.6745562 \\
\hline RM 337 & 144.766 & 383.20582 & 192 & 2 & 0 & 8 & 5 & 7 & 4 & 6 & 0.7913295 & 0.7385204 & 0.8896982 & 0.71875 & 0.6449704 \\
\hline RM 339 & 136.11499 & 194.5526 & 148 & 1 & 0 & 8 & 5 & 5 & 3 & 4 & 0.7153725 & 0.6938776 & 0.5296566 & 0.40625 & 0.4763314 \\
\hline RM 341 & 124.84082 & 251.62117 & 172 & 2 & 0 & 8 & 8 & 4 & 3 & 4 & 0.7204444 & 0.8545918 & 0.6181061 & 0.59375 & 0.5680473 \\
\hline RM 505 & 173.52414 & 249.29178 & 199 & 3 & 0 & 7 & 5 & 6 & 1 & 2 & 0.484241 & 0.6964286 & 0.9677419 & 0 & 0.0739645 \\
\hline RM 530 & 137.17836 & 177.19601 & 161 & 2 & 1 & 7 & 3 & 6 & 2 & 4 & 0.7032967 & 0.3137755 & 0.7700312 & 0.375 & 0.3920118 \\
\hline RM 569 & 136.86345 & 194.98306 & 175 & 2 & 0 & 8 & 6 & 7 & 3 & 6 & 0.7557058 & 0.7882653 & 0.760666 & 0.53125 & 0.6449704 \\
\hline Sum & & & & 51 & 27 & 182 & 118 & 154 & 66 & 113 & 17.1751 & 15.006378 & 18.41103 & 12.578125 & 13.214497 \\
\hline Average & & & & 2.21 & 1.17 & 7.91 & 5.13 & 6.69 & 2.87 & 4.91 & 0.7467435 & 0.6524512 & 0.8004796 & 0.546875 & 0.5745433 \\
\hline
\end{tabular}


RM505 to 0.882 in RM 149. For the check rice germplasms the average PIC value was 0.547 per marker. The range of PIC value was 0.0 in RM 505 to 0.812 in RM 251.

\section{Rare alleles}

A total of 51 rare alleles were identified from the 23 polymorphic loci. The highest number of rare alleles (6) was generated by the marker RM250 followed by RM207 (4 rare allele) and RM149, RM210, RM223, RM284 and RM505 generated 3 rare alleles each. Among the landraces, Kelas a non aromatic landrace from West Bengal had 7 rare alleles which is the maximum number in this set of rice accessions. Bangalaxmi and Bangladeshi Patnai, both non aromatic landraces from West Bengal have 5 rare alleles. Among the aromatic rice, the landrace Tulsimanjari from West Bengal, and the check Taraori Basmati had 4 rare alleles each. Chinikamini 2 and Narayanpurna, both aromatic landraces from West Bengal had 3 rare alleles each. Among the landraces from North East Indian States, Biroi dhan had 4 rare alleles followed by Aijung, Kalo Jeera, IC524502 and Morianghou with 3 rare alleles each.

\section{Null alleles}

Twenty seven null alleles were detected from 14 out of the 23 polymorphic loci. The highest number of null alleles (5 alleles) was generated by the marker RM80, followed by RM42 and RM207 with 4 null alleles each. Among the landraces, Kelas, a non aromatic landrace from West Bengal, had 4 null alleles, which is the highest number in this set of rice accessions. Bangalaxmi and Raghusail, both non aromatic landraces from West Bengal, and IC524531 from Nagaland had 3 null alleles each.

\section{Clustering of the rice genotypes}

The dendrogram given in Figure 2 was made from genetic similarity values. The strength of dendrogram nodes was estimated with a bootstrap analysis using 1000 permutations. The similarity among the rice accessions ranged from $8 \%$ to $64 \%$. At $8 \%$ level of similarity the dendrogram showed 2 clusters "A" and "B" with additional sub clusters within each. The cluster $B$ contained only four rice accessions, Bangalaxmi, Bangladeshi Patnai, Khandagiri and Sada Sankar; all non-aromatic landraces from West Bengal. The rest 87 accessions are grouped in cluster "A". At 10.8\% level of similarity cluster "A" divided into two sub clusters, of which one, cluster " $\mathrm{X}$ ", consisted predominantly of aromatic rice accessions from West Bengal and the other, cluster "Y", consisted predominantly of accessions from North Eastern States. The non aromatic rice accessions

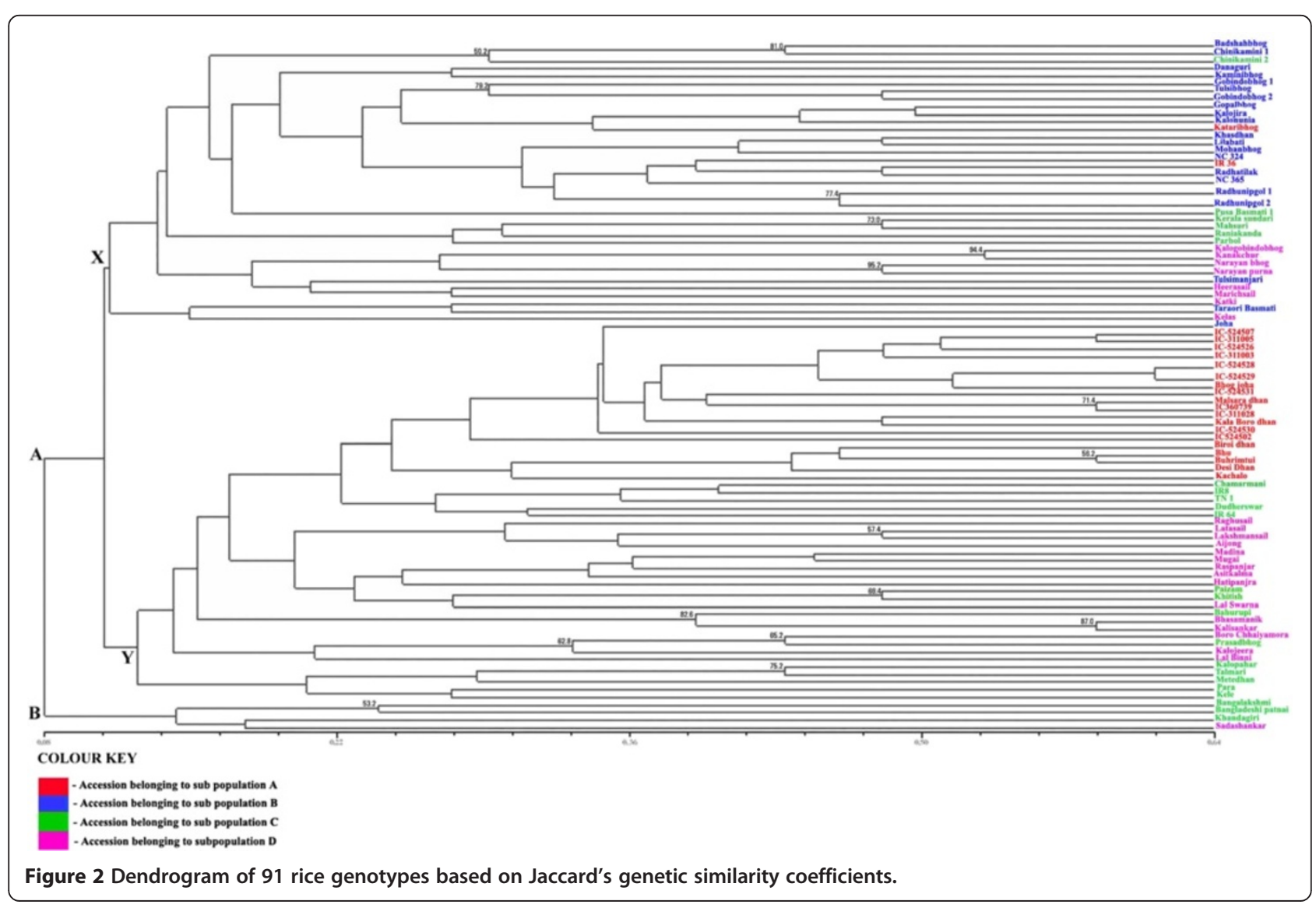


from West Bengal and the check genotypes were interspersed within the two predominant clusters.

The aromatic cluster " $\mathrm{X}$ " consisted of a total of 37 rice genotypes. The set included 27 aromatic landraces from West Bengal, 8 non aromatic landraces from West Bengal, and 3 check genotypes (IR36, Pusa Basmati 1 and Taraori Basmati). Within this cluster Kalogobindobhog and Kanakchur were 53\% similar and the two accessions of Radhunipagol, one each from repositories at Chinsurah and Phulia were $64 \%$ similar between themselves. The cluster was subdivided into two prominent sub clusters at about $11 \%$ level of similarity after which it segregated repeatedly to form sub clusters at progressively higher level of similarity until $64 \%$ level of similarity beyond which it segregated no more. Super imposition of the results of population structure analysis showed that the whole of sub population B was included in this cluster along with 8 members from sub population D. Six members from sub population $\mathrm{C}$ and 2 from sub population A were also included.

The predominant North Eastern cluster "Y" consisted of 50 different rice accessions out of which 12 were from Assam, 10 accessions from Nagaland, 1 from Manipur, 3 from Mizoram, 19 non-aromatic landraces from West
Bengal and 5 check genotypes. The cluster included the 2 accessions having the lowest amylose content (Lal Binni and Kalo Boro Dhan from Assam). The most similar accessions included IC524528 and IC524529 from Nagaland (64\% similar), and Bhu and Buhrimtui from Mizoram (58.4\% similar). Accessions of sub population A formed the majority in this cluster followed by accessions of sub population D and C. At 13.6\% level of similarity a sub cluster consisting of five non-aromatic landraces from West Bengal separated out. The genotypes in this group Kalopahar, Metedhan, Talmari, Kele and Para, had extra long to medium kernel length, medium kernel length and intermediate amylose content and were included in sub population C. There were no segregations beyond $64 \%$ level of similarity.

\section{Population structure analysis}

The inferred population structure is given in Figure 3. Admixture model-based simulations were carried out by varying $K$ from 2 to 10 with 10 iterations using all 91 genotypes which showed evident knees at $\mathrm{K}=4$ (Additional file 1: Figure S1 and Additional file 2: Figure S2) and were assigned to the corresponding A-D sub-populations representing $23.0 \%$ of A (21), $25.3 \%$ of B (23), $25.3 \%$ of C

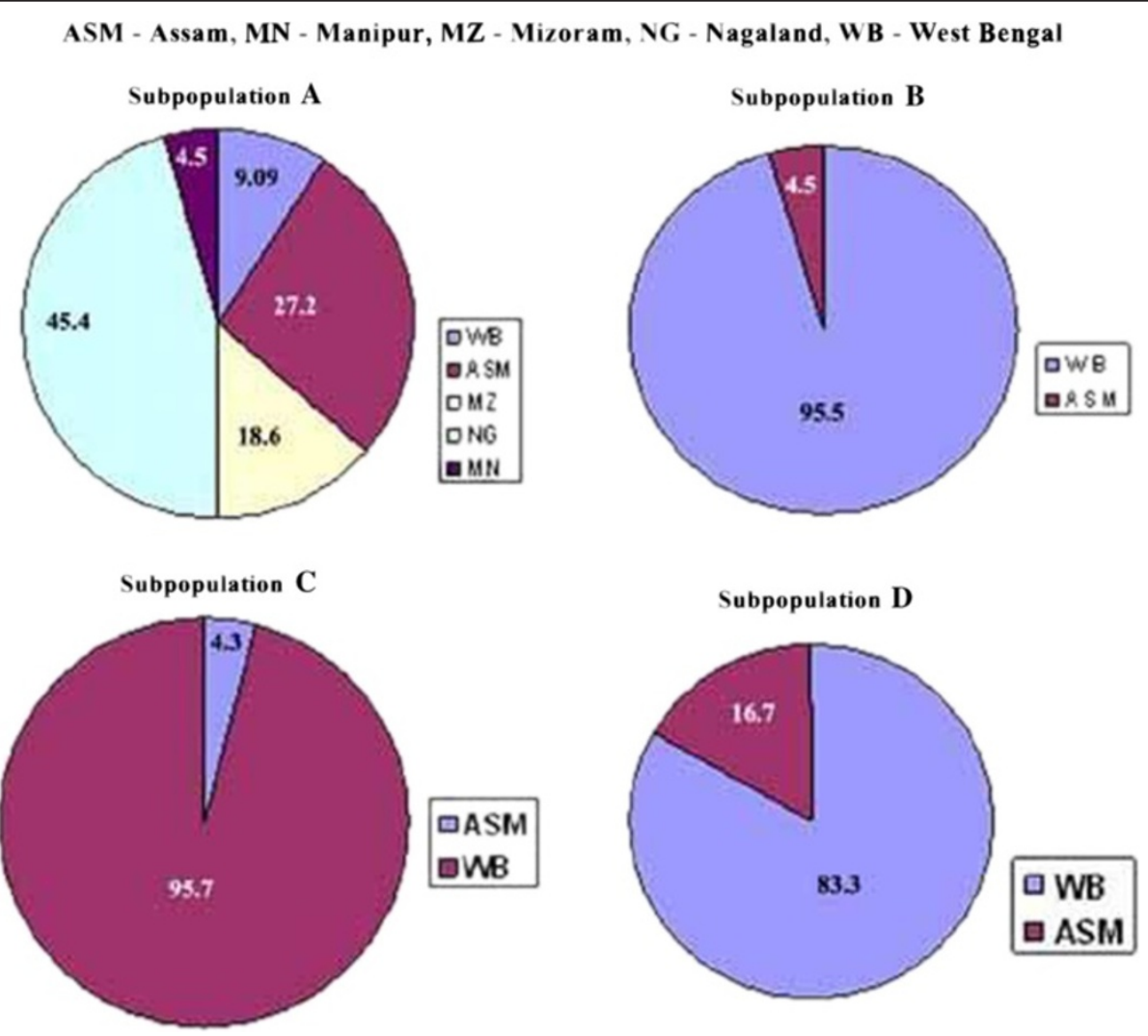

Figure 3 Pie diagram showing frequencies of individuals belonging to the four (A-D) structure group based on sampling area. 
(23) and $26.4 \%$ of D (24) used for the structure analysis. Genetic variation with subpopulations was tested using Fst statistics. The four sub-populations (A-D) had an Fst value of $0.398,0.364,0.206$ and 0.281 , respectively, with an average value 0.312 , indicating moderate population structure (Figure 3). These are population specific Fst value (not pair-wise Fst values between subpopulation) for 4 sub-populations (A-D) calculated using STRUCTURE software during construction of population structure. Based on sampling area frequencies of individuals belonging to each Structure group a pie diagram is given in Figure 3. Pair-wise Fst among the four sub-populations and their level of significance are given in Table 4.

The subpopulation A consisted of 22 accessions out of which 20 were from the North Easetrn States along with Kataribhog - an aromatic accession from West Bengal and IR36 - a high yielding check accession. They have extra long to medium grain length, predominantly medium kernel shape and intermediate amylose content. However the accession (Kalo Boro Dhan of Assam) with lowest amylose content was included in this group. The average number of alleles was 5.39 alleles / SSR marker. The prominent accessions having rare alleles were Biroi Dhan with 4, and Aijung, IC524502 and Morianghou with 3 rare alleles each.

The subpopulation B consisted of 22 aromatic accessions out of which 20 were from West Bengal, along with Joha from Assam and Taraori Basmati, a traditional basmati used as check in this study. This group was dominated by small kernel length, mostly medium kernel shape and low amylose content. The average number of alleles was 5.91 alleles / SSR marker. The prominent accessions having rare alleles were Tulsimanjari and Taraori Basmati, each with 4 rare alleles. There were 5 null alleles in this subpopulation wherein the accessions Danaguri and Lilabati have 2 and Badshahbhog with 1 null allele.

The subpopulation $\mathrm{C}$ consisted of 23 accessions which included 16 were non aromatic and 1 aromatic accessions from West Bengal, along with Prasasdbhog from Assam and 5 accessions used as check in this study. This group was dominated by extra long kernel length, medium

Table 4 Pair-wise FST estimation among four subpopulations estimated using Arlequin

\begin{tabular}{ccccc}
\hline Populations & \multicolumn{4}{c}{ Populations groups } \\
\cline { 2 - 5 } & A & B & C & D \\
\hline A & - & 0.20 & 0.14 & 0.16 \\
B & $0.22^{* *}$ & - & 0.15 & 0.14 \\
C & $0.19^{*}$ & 0.15 & - & $0.10^{*}$ \\
D & 0.17 & 0.15 & $0.08^{* *}$ & - \\
\hline
\end{tabular}

*Significance at $\mathrm{P}<0.05$ at 1,000 permutations.

** Significance at $P<0.01$ at 1,000 permutations. kernel shape and intermediate to high amylose content. The average number of alleles was 8.22 alleles / SSR marker. The prominent accessions with rare alleles were Bangalaxmi and Bangladeshi Patnai, each with 5 rare alleles. There were 8 null alleles in this subpopulation wherein the accessions Bangalaxmi had 3 Chamormoni and Metedhan had 2 and Para had 1 null allele.

The subpopulation D consisted of 24 accessions which included 16 were non aromatic and 4 aromatic accessions from West Bengal, along with 4 accessions from Assam. This group was characterized by small to extra long kernel length, round to slender kernel shape and very low to high amylose content. The average number of alleles was 7.35 alleles / SSR marker. The prominent accessions with rare alleles were the non aromatic accession from West Bengal, Kelas with 7 and Heerasail and Sada Sankar with 3 rare alleles each. There were 7 null alleles in this subpopulation wherein the accessions Kelas had 4 and Raghusail had 3 null alleles.

\section{Discussion}

This study calculates the genetic distances among accessions of 83 landraces from West Bengal and the North Eastern States and 8 check accessions using 23 SSR markers and examines the population structure among the accessions using model-based clustering approach. The set included both aromatic and non aromatic accessions. The 23 SSR markers used for this study were mapped previously [45]. All the SSR markers used for this study revealed a clear and consistent amplification profile. The results are consistent with published reports on microsatellite frequency in the rice genome [46]. Stutter bands, which are minor products amplified in PCR that have lower intensity than the main allele and normally lacks or has extra repeat units [37] were also present in the profiles of most of the markers used. Null alleles were present probably due to mutations in the binding region of one or both of the microsatellite primers, thereby inhibiting primer annealing [37]. The number of alleles ranged from 2 in RM112 to 13 in RM 149 and the average was 7.9 alleles / locus. This number is higher than that reported for average number of alleles by Cho (5.5 alleles/locus) [36] or Yu et al., (6.3 alleles/locus) [46]. This is also smaller than the study by Yang et al., [15] which reported 9.3 alleles/ locus. However the average number of allele per locus for this study is comparable to reports of 7.8 alleles /locus by Jain et al., [47]; as recorded from a set of Indian aromatic and quality rice accessions. From the PIC values it is evident that within the landraces the allelic diversity is the highest among the non aromatic germplasm accessions from West Bengal followed by the aromatic accessions from the same state. The allelic diversity of the accessions from the North Eastern States stands third for this experimental set of rice accessions. 
Most of the markers were selected from chromosome 8 and two important traits of aromatic and basmati rice, aroma and cooked kernel elongation ratio [42], had been mapped earlier using RFLP markers to chromosome eight. In the international market there is a huge demand for long, slender rice kernels which elongate to twice or more the original length. One of the aims of the study was to find out whether the chosen set of rice could be segregated into various categories according to their length and shape of their kernels. The clustering of the rice genotypes according to the SSR profiles however was not able to distinctly classify the set into small, medium, long or extra long length groups. This discrepancy was perhaps due to the fact that the loci involved in this study reveal only a small part of the genotype/phenotypes association of otherwise complex traits like grain and kernel length and shape, which are actually attributed to multiple loci with small effect $[24,25]$.

The analysis of the SSR profiles of all the aromatic landraces from West Bengal and Assam and check basmati rice revealed that there were 13 rare alleles each within the set of 27 aromatic landraces of West Bengal and 6 aromatic landraces Assam. The two check basmati genotypes had 5 rare alleles. Clearly the diversity present in the Assam landraces are of a higher magnitude. Also in the dendrogram in Figure 2 Assam landraces were grouped in a separate cluster from the rest of the aromatic landraces and Basmati rice indicating a difference in their origin. Inclusion of a substantial number of Basmati genotypes would perhaps given rise to a separate cluster for them since the distinctiveness of Basmati from indigenous aromatic rice has already been proved by previous workers [48-51].

Of the total number of different alleles identified, the highest (154 alleles) was found in the non aromatic landraces from West Bengal. That set also had the highest average PIC value/marker. One of the non aromatic genotypes Kelas had 7 rare and 4 null alleles. In addition the accessions Bangalaxmi and Bangladeshi Patnai, had 5 rare alleles each. This category of rice has been subjected to lesser human selection as compared to the aromatic genotypes. The aromatic genotypes of West Bengal have been specifically selected for traits like slender kernels, non sticky cooked kernels, shorter cooking time and strong aroma thereby narrowing their genetic base. Their end use is also limited to making desserts, as quality table rice and as offerings in Hindu ceremonies. The non aromatic rice genotypes on the other hand has undergone lesser selection pressure and are used for a variety of purposes like making of table rice, puffed rice, popped rice, beaten rice, rice flakes etc. Some of them like Kabirajsail (not included in this study) are reported to have medicinal values and some others like Raghusail, Bhasamanik and Kelas are reported to have resistance against bacterial leaf blight [52].
From the total number of alleles (113 alleles) and the average PIC values $(0.5745 /$ marker $)$ it is evident that the genetic diversity in the genotypes from the North Eastern States were far lesser than the diversity of genotypes from West Bengal. Interestingly within the 26 North Eastern rice genotypes, the 12 genotypes from Assam had 94 alleles and an average PIC value of $0.6581 /$ marker. The rest of the North East accessions, of which majority were from Nagaland, had 71 alleles and an average PIC value of $0.4214 /$ marker. The topography of Assam is characterized by both hills and plains with an altitude ranging from $43 \mathrm{~m}-1736 \mathrm{~m}$ and a climate type from tropical to subtropical. The hilly areas of Karbi Anglong and North Cachar Hills are dominated by "jhoom" (slash and burn method) cultivation while the plains of Assam are dominated by lowland and deep water rice cultivation [6]. On the other hand the states of Manipur, Mizoram and Nagaland are characterized by a subtropical to temperate type of climate, with an average land elevation ranging from $235 \mathrm{~m}$ to $2745 \mathrm{~m}$ and the rice cultivation is mostly upland type [6]. The more diverse nature of the Assamese landraces may be a reflection of the prevalent diverse agroclimatic conditions of the State. However, further collection and characterization of more landraces are required to establish this association.

The population structure analysis revealed 4 subpopulations A, B, C and D out of which the majority of accessions were included in subpopulation $\mathrm{D}$. The groupings of accessions obtained using structure analysis are well in agreement with the distance-based clustering. In the dendrogram the accessions of subpopulations " $A$ " and "B" were far less scattered than the accessions of subpopulations $C$ and $D$. The kernel length, kernel shape, amylose content and aroma levels of the accessions of subpopulation A were distinctly different from that of subpopulation B. Subpopulation "A" consisted mainly of accessions from the North Eastern States and subpopulation "B" consisted of aromatic accession from West Bengal. Hence some kind of geographical grouping was evident between these two subpopulations. However, in subpopulations C and D, which consisted mainly of non aromatic accessions from West Bengal and the check genotypes with more or less similar kernel morphology; no geographical grouping was obvious.

\section{Conclusion}

Each of the accessions could be identified unequivocally by the SSR profiles. There are obvious differences between the aromatic and non aromatic landraces and the check genotypes, which are evident in the dendrogram. Genetically the non-aromatic landraces from West Bengal were most diverse followed by the aromatic landraces from the same state. The North Eastern accessions ranked third. Further, grouping of accessions based on their agronomic 
traits may serve as a resource for future studies, leading to the improvement of rice. Although the Basmati rice fetches a premium in the international market due to consumer quality preference of the West, it is a traditional observation that the aromatic landraces are more versatile. They are more soothing to the palate and can be used for a variety of cuisine starting from table rice to desserts, from offerings in various from religious ceremonies to diet for the convalescent and for making popped rice. Basmati accessions on the other hand can be used only for making certain rice delicacies and can never be used for other purposes. The landraces, whether aromatic or not, were confirmed to be adapted to the agro climatic conditions of their respective place of cultivation. Interestingly, Tulaipanji, an aromatic accession originally cultivated in the cooler northern districts of the state of West Bengal, India, was found to lose its aroma when cultivated in the warmer southern districts [52]. Some of these aromatic landraces are also resistant to biotic stress, for example Kataribhog is resistant to rice Tungro virus [52]. The quality of straw produced by the landraces is more suitable for thatching huts [52]. Moreover, the landraces are the means of sustenance for the marginal and impoverished tribal farmers. In-situ preservation of the landraces found in the biodiversity hotspots is also a means of protection of the culture, heritage and socio economic structure of the farmers' population of those places.

\section{Additional files}

Additional file 1: Figure S1. Inferred population structure. K varying from 2 to 10 with 10 iterations.

Additional file 2: Figure S2. Optimization of K (number of populations) value in population genetic structure using log probability data Ln (K) as function based on Island model (IM) of microsatellite markers (Evanno et al. 2005). The population structure among rice genotypes was found most suitable at $K=4$ with $\operatorname{Ln}(K)$ value of -10206 .

\section{Competing interests}

The authors declare that they have no competing of interests.

\section{Authors' contributions}

$\mathrm{BD}$ did all the experiments pertaining to DNA extraction, PCR, PAGE, collected data and was involved in data analysis and drafting of the manuscript. SS procured the rice accessions from various repositories of the North Eastern States, did some of the experimentation pertaining to PCR and PAGE and helped with data collection and analysis. BR helped with maintenance of the rice accessions. MG procured the rice accessions from various repositories of West Bengal and helped with the experimentation. MP and SKP did the bootstrap analysis and population structure analysis and analysis with arlequin. TKG was involved with the conception of the work and gave the final approval to the version of the manuscript that is being sent for consideration for publication. All authors read and approved the final manuscript.

\section{Acknowledgement}

The authors wish to thank National Bureau of Plant Genetic Resources, India, Assam Agriculture University, Agricultural Training Centre Fulia, Rice Research Station Chinsurah, and State Agricultural Research Farm Kashipur for providing us with rice accessions. They also wish to thank the Department of science and Technology for providing the research funding through Bose Institute and for providing the fellowship to Basabdatta Das. We also thank the University Grants Commission for providing fellowship to Samik Sengupta through Calcutta University.

\section{Author details}

${ }^{1}$ Division of Plant Biology, Bose Institute, Main Campus, 93/1 A.P.C. Road, 700009 Kolkata, West Bengal, India. ²Department of Horticulture, Institute of Agricultural Science, University of Calcutta, 35, Balligunge Circular Road, 700029 Kolkata, West Bengal, India. ${ }^{3}$ Department of Agronomy, Bidhan Chandra Krishi Viswavidyala Mohanpur, District - Nadia - 741252, Kolkata, West Bengal, India. ${ }^{4}$ National Institute of Plant Genome Research (NIPGR), Aruna Asaf Ali Marg, 110067 New Delhi, India.

Received: 17 April 2012 Accepted: 12 August 2013 Published: 15 August 2013

\section{Reference}

1. Londo JP, Chiang YC, Hung KH, Chiang TY, Schaal BA: Phylogeography of Asian wild rice, Oryza rufipogon, reveals multiple independent domestications of cultivated rice. Oryza sativa. PNAS 2006, 103(25):9578-9583.

2. Bautista NS, Solis R, Kamijima O, Ishii T: RAPD, RFLP and SSLP analyses of phylogenetic relationships between cultivated and wild species of rice Genes. Genet Syst 2001, 76(2):71-79.

3. Second G: Origin of the genic diversity of cultivated rice (Oryza spp.): Study of the polymorphism scored at 40 isozyme loci Jpn. J Genet 1982, 57:25-57.

4. Khush GS: Origin, dispersal, cultivation and variation of rice. Plant Mol Biol 1997, 35:25-34.

5. Harlan JR: Crops and Man. Madison, Wisconsin: American Society of Agronomy and Crop Science Society of America; 1975.

6. Hore DK: Rice diversity collection, conservation and management in northeastern India. Genet Resour Crop Evol 2005, 52:1129-1140.

7. Mackill DJ: Plant genetic resources. Classifying japonica rice cultivars with RAPD markers. Crop. Sci. 1995, 35:889-894.

8. Glaszmann JC: Isozymes and classification of Asian rice varieties. Theor Appl Genet 1987, 74:21-30.

9. Nei M, Li W: Mathematical model for studying genetic variation in terms of restriction endonucleases. Proc Natl Acad Sci U S A 1979, 76:5269-5273

10. Wu KS, Tanksley SD: Abundance, polymorphism and genetic mapping of microsatellites in rice. Mol Gen Genet 1993, 241:225-235.

11. Panaud O, McCouch SR, Chen X: Development of microsatellite markers and characterization of simple sequence length polymorphism (SSLP) in rice (Oryza sativa L.). Mol Gen Genet 1996, 252:597-607.

12. Xiao J, Li J, Yuan L, McCouch SR, Tanksley SD: Genetic diversity and its relationship to hybrid performance and heterosis in rice as revealed by PCR based markers. Theor App/ Genet 1996, 92:637-643.

13. Olufowote JO, Xu Y, Chen X, Park WD, Beachell HM, Goto M, McCouch SR: Comparative evaluation of within cultivar variation of rice (Oryza sativa L.) using microsatellite and RFLP markers. Genome 1997, 40:370-378.

14. Thanh ND, Zheng HG, Dong NV, Trinh LN, Ali ML, Nguyen HT: Genetic variation in root morphology and microsatellite DNA loci in upland rice (Oryza sativa L.) from Vietnam. Euphytica 1999, 105:43-51.

15. Yang GP, Maroof MAS, Xu CG, Zang Q, Baiyashev RM: Comparative analysis of microsatellite DNA polymorphism in landraces and cultivars of rice. Mol Gen Genet 1994, 245:187-194.

16. Akagi K, Sandig V, Vooijs M, Van der Valk M, Giovannini M, Strauss M, Berns A: Pre-mediated somatic site-specific recombination in mice. Nucl Acids Res 1997, 25:1766-1773.

17. Blair MW, Panaud O, McCouch SR: Inter simple sequence repeat (ISSR) amplification for microsatellite motif frequency and fingerprinting in rice (Oryza sativa L.). Theor Appl Genet 1999, 98:780-792.

18. Joshi SP, Gupta VS, Aggarwal RK, Ranjekar PK, Brar DS: Genetioc diversity and phylogenetic relationship as revealed by inter simple sequence repeat (ISSR) polymorphism in the genus Oryza. Theor App/ Genet 2000, 100:1311-1320.

19. Bobba S, Siddiq EA: ISSR and SSR markers based on AG and GA repeats delineate geographically diverse (Oryza nivara L.) accessions and reveal rare alleles. Curr Sci 2003, 84:683-689. 
20. Herrera TG, Duque DP, Almeida IP, Núñez GT, Pieters AJ, Martinez CP, Tohme $J$ M: Assessment of genetic diversity in Venezuelan rice cultivars using simple sequence repeats markers. Electron J Biotechnol 2008, 11(5):1-14

21. Pervaiz ZH, Rabbani MA, Khaliq I, Pearce SR, Malik SA: Genetic diversity associated with agronomic traits using microsatellite markers in Pakistani rice landraces. Electron J Biotechnol 2010, 13(3):1-14.

22. Maytinee Kladmook M, Kumchoo T, Hongtrakul V: Genetic diversity analysis and subspecies classification of Thailand rice landraces using DNA markers. Afr J Biotechnol 2012, 11(76):14044-14053.

23. Behera L, Patra BC, Sahu RK, Nanda A, Sahu SC, Patnaik A, Rao GJN, Singh ON: Assessment of genetic diversity in medicinal rices using microsatellite markers. Australian Journal of Crop Science 2012, 6(9):1369-1376.

24. Ge XJ, Xing YZ, Xu CG, He YQ: QTL analysis of cooked rice grain elongation, volume expansion, and water absorption using a recombinant inbred population. Plant Breeding 2005, 124:121-126.

25. Bai X, Luo L, Yan W, Rao MK, Zhan W, Xing Y: Genetic dissection of rice grain shape using a recombinant inbred line population derived from two contrasting parents and fine mapping a pleiotropic quantitative trait locus qGL7. BMC Genetics: 2010, 11:16.

26. Ahmadi J, Fotokian MH: Identification and mapping of quantitative trait loci associated with salinity tolerance in rice (Oryza Sativa) using SSR markers. Iranian Journal of Biotechnology 2011, 9:1.

27. Lin MH, Lin CW, Chen JC, Lin YC, Cheng SY, Liu TH, Jan FJ, Wu ST, Thseng FS, Ku HM: Tagging Rice Drought-related QTL with SSR DNA Markers. Crop, Environment \& Bioinformatics 2007, 4:65-76.

28. Sudharshan IN, MK Prasad MK, Pandey MK, Shobha Rani N: Historical significance, grain quality features and precision breeding for improvement of export quality basmati varieties in India. Indian. J Crop Science 2006, 1(1-2):29-41.

29. Biswas S, Dey N, Ray Chaudhury T, Dey SR, De M, Prasad M, Ghose TK: Indentification of major QTL for cooked kernel elongation on chromosome 8 of rice (O.sativa L). Hyderabad, India: Proceedings of Symposium on Comparative and Functional Genomics; 2004:31.

30. Eizenga GC, Agrama HA, Lee FN, Yan W, Jia Y: Identifying Novel Resistance Genes in Newly Introduced Blast Resistant Rice Germplasm. Crop Sci 2006, 46:1870-1878.

31. Breusegem FV, Dekeyser R, Garcia AB, Claes B, Gielen J, Van Montagu M, Caplan AB: Heat-inducible rice hsp82 and hsp70 are not always coregulated. Planta 1994, 193(1):57-66.

32. Standard Evaluation Systems for rice: International Rice Testing Program. 4th edition. Manila, Philippines: IRRI; 1996.

33. Das B: Genetic diversity analysis of a set of rice landraces using dna markers. PhD thesis. Jadavpur University: Llfe Science Department; 2012.

34. Walbot V: Preparation of DNA from single rice seedling. Rice Genet News/ 1988, 5:149-151.

35. Sambrook J, Fritsch EF, Maniatis T: Molecular cloning: A laboratory manual. 2nd edition. New York: Cold Spring Harbour Laboratory Press; 1989.

36. Cho YG, Ishii T, Temnykh S, Chen X, Lipovich L, Park WD, Ayres N, Cartinhour S, McCouch SR: Diversity of microsatellites derived from genomic libraries and GenBank sequences in rice (Oryza sativa L.). Theor App/ Genet 2000, 100:713.

37. Callen DF, Thompson AD, Shen Y, Phillips HA, Richards Rl, Mulley JC, Sutherland GR: Incidence and origin of "null" alleles in the (AC) n microsatellite markers. Am J Hum Genet 1993, 52:922-927.

38. Jain S, Jain RK, McCouch SR: Genetic analysis of Indian aromatic and quality rice (Oryza sativa L.) germplasm using panels of fluorescentlylabeled microsatellite markers. Theor Appl Genet 2004, 109:965-977.

39. Jaccard P: Nouvelle recherches sur la distribution florale. Bulletin de la Socie'te' Vaudoise des Sciences Naturelles 1908, 44:223-270.

40. Rohlf FJ: NTSYS-pc. Numerical taxonomy and multivariance analysis system version 2.02e. New York, USA: Exeter Software; 1997. http://www. exetersoftware.com/cat/ntsyspc/ntsyspc.html.

41. Yap IP, Nelson R, Boot W: A programm for performing boot strap analysis of binary data to determine the confidence limits of UPGMA-based dendrograms. IPRI Discussion Paper series; 1995.

42. Anderson PA, Lawrence GJ, Morrish BC, Ayliffe MA, Finnegan EJ, Ellis JG: Inactivation of the Flax rust resistance gene $M$ associated with loss of a repeated unit within the leucine rich repeat coding region. Plant Cell 1997, 9:641-651.

43. Pritchard JK, Stephens M, Donnelly P: Inference of population structure using multilocus genotype data. Genetics 2000, 155:945-959.
44. Excoffier L, Lischer HEL: Arlequin suite ver 3.5: A new series of programs to perform population genetics analyses under Linux and Windows. Molecular Ecology Resources 2010, 10:564-567.

45. McCouch SR, Teytelman L, Xu Y, Lobos KB, Clare K, Walton M, Fu B, Maghirang R, Li Z, Xinfg Y, Zhang Q, Kono I, Yano M, Fjellstorm R, DeClerck G, Schneider D, Cartinhour S, Ware D, Stein L: Development and mapping of 2240 new SSR markers for rice (Oryza sativa L.). DNA Res 2002, 9:199-207.

46. Yu SB, Xu WJ, Vijayakumar CHM, Ali J, Fu BY, Xu JL, Jiang YZ, Marghirang R, Domingo J, Aquino C, Virmani SS, Li ZK: Molecular diversity and multilocus organization of the parental lines used in the International Rice Molecular Breeding Program. Theoretical and Applied Genetics 2003, 108:131-140.

47. Jain N, Jain S, Saini N, Jain RK: SSR analysis of chromosome 8 regions associated with aroma and cooked kernel elongation in Basmati rice. Euphytica 2006, 152:259-273.

48. Nagaraju J, Kathirvel M, Kumar RR, Siddiq EA, Hasnain SE: Genetic analysis of traditional and evolved Basmati and non Basmati rice varieties by using fluorescence based ISSR PCR and SSR markers. Proc Natl Acad Sci, USA 2002, 99:5836-5841.

49. Archak S, Lakshminarayanareddy V, Nagaraju J: High-throughput multiplex microsatellite marker assay for detection and quantification of adulteration in Basmati rice (Oryza sativa). Electrophoresis 2007, 28:2396-2405.

50. Rashid M, Ren-hu L, Wei J, Yong-han X, Fu-lin W, Yue-zhi T, Jun-mei W, Cheema AA, Jin-qing C, He G: Genomic diversity among Basmati rice (Oryza sativa $\mathrm{L}$ ) mutants obtained through $60 \mathrm{Co}$ gamma radiations using AFLP markers. Afr J Biotechnol 2009, 8(24):6777-6783.

51. Singh D, Kumar A, Sirohi A, Kumar $P$, Singh J, Kumar V, Jindal A, Kumar $S$, Kumar N, Kumar V, Sharma V, Gupta S, Gupta S: Improvement of Basmati rice (Oryza sativa L.) using traditional breeding technology supplemented with molecular markers. Afr J Biotechnol 2011, 10(4):499-506

52. Deb D: Folk Rice varieties of West Bengal: agronomic and morphological characteristics. New Delhi: Research Foundation for Science, Technology and Ecology; 2000

doi:10.1186/1471-2156-14-71

Cite this article as: Das et al: Genetic diversity and population structure of rice landraces from Eastern and North Eastern States of India. BMC Genetics 2013 14:71.

\section{Submit your next manuscript to BioMed Central and take full advantage of:}

- Convenient online submission

- Thorough peer review

- No space constraints or color figure charges

- Immediate publication on acceptance

- Inclusion in PubMed, CAS, Scopus and Google Scholar

- Research which is freely available for redistribution 